\title{
Comparison of Global Nonlinear Models and "Model-on-Demand" Estimation Applied to Identification of a RTP Wafer Reactor
}

\author{
M.W. Braun, D.E. Rivera, A. Stenman, W. Foslien \\ Department of Electrical Engineering \\ Linköping University, SE-581 83 Linköping, Sweden \\ URL: http://www. control.isy.liu.se \\ Email: stenman@isy.liu.se
}

September 1, 1999

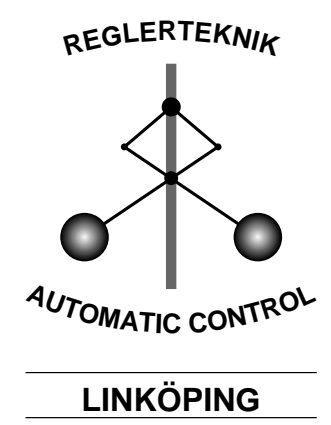

Report no.: LiTH-ISY-R-2184

To be presented at the 38th IEEE Conference on Decision and Control, Phoenix, Arizona, USA, December 7-10, 1999.

Technical reports from the Automatic Control group in Linköping are available by anonymous ftp at the address ftp.control.isy.liu.se. This report is contained in the PDF file 2184.pdf. 


\title{
Comparison of Global Nonlinear Models and "Model-on-Demand" Estimation Applied to Identification of a RTP Wafer Reactor
}

\author{
M. W. Braun ${ }^{\dagger}$, D. E. Rivera ${ }^{\dagger 1}$, A. Stenman ${ }^{\dagger \dagger}$, and W. Foslien ${ }^{\dagger \dagger}$ \\ ${ }^{\dagger}$ Department of Chemical, Bio, and Materials Engineering \\ Control Systems Engineering Laboratory, Manufacturing Institute \\ Arizona State University, Tempe, Arizona 85287-6006 \\ ${ }^{\dagger}$ Division of Automatic Control, Department of Electrical Engineering \\ Linköping University, 5-581 83 Linköping, Sweden \\ t† Honeywell Technology Center \\ 3660 Technology Drive, Minneapolis, MN 55418
}

\begin{abstract}
"Model on Demand" (MoD) simulation of the temperature dynamics in a simulated Rapid Thermal Processing (RTP) reactor is compared against various types of global models (ARX, semiphysical, combined semiphysical with neural net). The identification data is generated from a m-level pseudo-random sequence input whose parameters are specified systematically using a priori information readily available to the engineer. The MoD estimator outperforms the ARX model and two semi-physical models, while matching the performance of a combined semi-physical with neural net model. This makes MoD estimation an appealing alternative to global methods because of its reduced engineering effort and simplified a priori knowledge regarding model structure.
\end{abstract}

\section{Introduction}

As advanced control methods rely more and more on nonlinear models for prediction, engineers are challenged to make efficient use of available a priori knowledge to develop useful models. Traditional treatments of the nonlinear identification problem usually involve global methods, such as neural networks, radial basis networks, wavelets, and other kinds of nonlinear parametric models. These models compress all available information into a compact model. However, when dealing with large sets of data, this approach becomes

\footnotetext{
${ }^{1}$ To whom all correspondence should be addressed. phone: (480) 965-9476 fax: (480) 965-0037; e-mail: daniel.rivera@asu.edu
}

less attractive to deal with because of the difficulties in specifying model structure, and the complexity of the associated optimization problem (which is usually highly nonconvex).

Inspired by ideals from local modeling and database systems technology, the Model-on-Demand (MoD) estimator takes a conceptually different point-of-view. In MoD estimation all observations are stored on a database, and the models are built "on demand" as the actual need arises. Local models such as the Model on Demand predictor use only small portions of data, relevant to the region of interest, to determine a model as needed. The variance/bias tradeoff inherent to all modeling is optimized locally by adapting the number of data and their relative weighting. The MoD approach enhances local modeling and provides the potential for performance rivaling that of global methods (such as neural networks) while involving less complex a priori knowledge from the user and providing more reliable numerical computations.

Rapid thermal processing (RTP) reactors are playing an increasingly important role in the semiconductor processing industries by offering wafer fabrication operations such as annealing, oxidation and chemical vapor deposition in succession, without having to move the wafer between reactors. These reactors operate over a wide range of temperatures, from 673 to $1573 \mathrm{~K}$, over which the properties of the wafer and the process capability of the reactor vary significantly, (Reid and Sitaram, [11]). Thus, RTP application provides a useful testbed for comparison of global and local nonlinear modeling approaches. 
Previous work derived "plant-friendly" control-relevant guidelines capable of producing a database rich enough to support MoD based simulation (Braun et al.[2]). More than one set of design variables met the guidelines. The dataset which provided the best validation for the MoD estimator was used in this work. This paper extends our previous work by providing a comparison of rival technologies utilizing a common estimation dataset.

The paper begins with a discussion of how to estimate values for design variables for multi-level pseudorandom sequence (m-level PRS) inputs. The theory behind the MoD predictor is discussed in Section 3 . Semi-physical modeling is covered in Section 4 and leads into the combination of semi-physical models and neural networks. Section 5 deals with the application of the theory to the RTP case study. Finally, we discuss the results of the case study with a comparison of the work required to develop the reduced semi-physical with neural network model and the MoD model. We also compare MoD estimation of the temperature dynamics in the RTP reactor simulation against the estimation by various types of global models (ARX, semiphysical, combined semiphysical with neural net).

\section{Multi-level Pseudo-Random Signal Design}

The theory behind the generation and application of mlevel PRS inputs in system identification is well developed [6]. m-level PRS signals are generated in a similar manner to the PRBS using shift registers and modulo addition. As with the PRBS, m-level PRS inputs are periodic, deterministic signals and have an autocorrelation function similar to white noise. Recent work by Barker and coworkers develops theoretical methods for generation and design of m-level PRS signals with suppressed harmonics of multiples 2, 3 and 5. [1].

The user of m-level PRS signals is faced with the task of determining values for the design variables needed to generate the signal. These include switching time $T_{s w}$, the number of shift registers $n_{r}$, the number of elements in the Galois Field $G F(q)$, the number of levels of the signal $m$, the harmonics to be suppressed $h$ and the signal amplitude. The algorithms for generating these signals is covered in Godfrey [6] and in Barker [1]. This section describes how to use the following $a$ priori knowledge to arrive at values for the design variables: Desired closed-loop speed of response, estimated range of dominant time constant, order of nonlinearity of plant and structure of model to be fit, and acceptable signal length and amplitude.

Figure 1 is a flowchart showing how a user may systematically determine the design variables to generate a m-level PRS signal. In some cases, the user may also have to ease the constraints of signal amplitude or signal length in order to meet the identification objectives. A priori knowledge of the dominant time con-

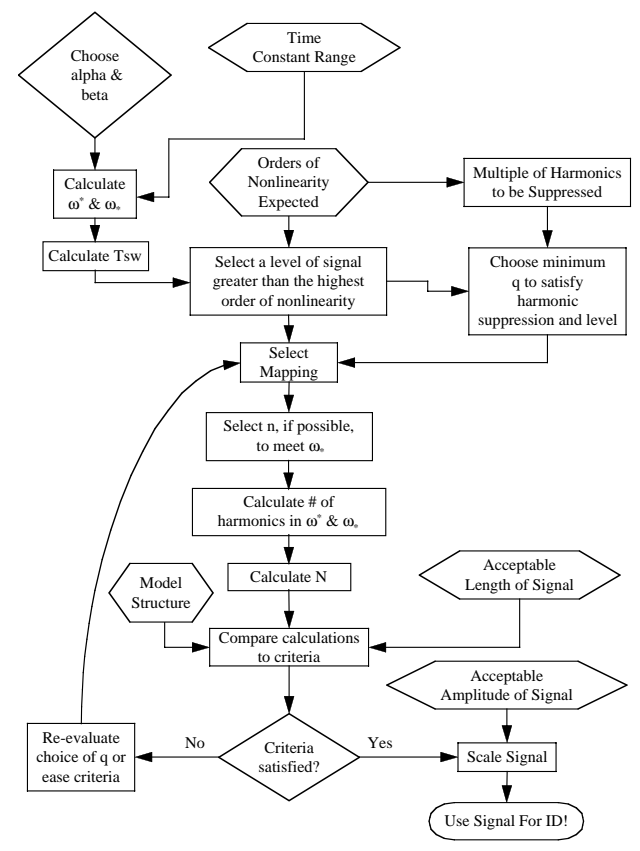

Figure 1: Flowchart for multi-level PRS signal design

stant range $\left(\tau_{d o m}^{H}\right.$ to $\left.\tau_{d o m}^{L}\right)$ makes it possible to place the power of the input signal in the frequency range important for control. The frequency range of interest (corresponding to that between the first harmonic and the bandwidth of the power spectrum) should fall within the range specified in Equation 1:

$$
\omega_{*}=\frac{1}{\beta \tau_{d o m}^{H}} \leq \omega \leq \frac{\alpha}{\tau_{d o m}^{L}}=\omega^{*}
$$

The parameters $\alpha$ and $\beta$ define the desired range for high and low frequency excitation. $\beta$ (normally equal to 3,4 , or 5) specifies low frequency information corresponding to the $95 \%$ settling time, $98 \%$ settling time, and $99 \%$ settling time, respectively of the open-loop plant. $\alpha$ determines the high frequency content in the signal and is defined on the basis of how much faster the intended closed-loop speed of response will be relative to open-loop. Typical values chosen for $\alpha$ and $\beta$ are 2 , and 3, respectively. However, as the plant of interest or operating region becomes more nonlinear, the frequency range of the input signal may not be as important as the shape of the input signal, as noted by Pearson and Ogunnaike [7]. $\alpha$ and the number of levels may be used to adjust the shape of the signal.

The number of levels $m$ should be at least one greater than the nonlinearity order in the candidate model. The size of the Galois field $q$ must support the required suppression of harmonics. Harmonics can be suppressed in multiples of $2(q \geq 3), 2$ and $3(q \geq 7)$, 
and 2,3 , and $5(q \geq 31)$. $q$ must always be greater or equal to the required number of levels $(q \geq m)$. Estimates of the switching time, $T_{s w}$, and number of shift registers, $n_{r}$, can be made from Equation 2 .

$$
T_{s w} \leq \frac{2.78}{\omega^{*}}, \quad \omega_{*} \geq \frac{2 \pi}{T_{s w}\left(q^{n_{r}}-1\right)}
$$

Harmonics suppression in m-level PRS signals will allow decomposition of the linear/nonlinear kernels. The degree of excitation corresponding to harmonic suppression can be found in Braun et al. [2].

The number of elements in one cycle of the input signal is determined by

$$
N_{c y c}=\left(q^{n_{r}}-1\right)
$$

$T_{c y c}=N_{c y c} * T_{s w}$ specifies the signal duration for one cycle. If signal duration represents a significant constraint in the input design problem, then the multiples of harmonics to be suppressed and the frequency range of the signal may have to be compromised in order to reduce the length of the signal.

Finally, it is important to choose a "plant-friendly" amplitude for the signal. Since an m-level PRS signals can switch from minimum to maximum values in one switching time, the acceptable amplitude span must be selected to avoid actuator restrictions, product quality constraints, move size restrictions, and so forth.

\section{Model-on-Demand Prediction}

"Just-In-Time" (JIT) or "Model on Demand" (MoD) modeling is a novel paradigm first proposed by $\mathrm{Cy}-$ benko [4]. The philosophy of MoD modeling is that a model is not estimated until it is really needed. All observations of the process are stored in a database, which is accessed to estimate a local model at the current operating point. The variance/bias tradeoff is optimized locally by adapting the number of data and their relative weighting. The MoD approach enhances local modeling and provides a reasonable alternative to nonlinear black-box techniques that optimize model predictions globally (such as neural networks). The MoD modeling formulation pursued in this paper follows from the approach of Stenman, Gustavsson, and Ljung [12] and Stenman [13].

Consider a SISO process with nonlinear ARX structure, i.e.,

$$
y(k)=m(\varphi(k))+e(k), k=1, \ldots, M,
$$

where $m(\cdot)$ is an unknown nonlinear mapping and $e(k)$ is an error term modeled as i.i.d. random variables with zero mean and variance $\sigma_{k}^{2}$. The MoD predictor attempts to estimate a value $\hat{y}$ based on a local neighborhood of the regressor space $\varphi(t)$ (Stenman [12]). The regressor vector is of the form

$\varphi(t)=\left[y(t-1) \ldots y\left(t-n_{a}\right) u\left(t-n_{k}\right) \ldots u\left(t-n_{b}-n_{k}\right)\right]^{T}$

where $n_{a}, n_{b}$, and $n_{k}$ denote the number of previous outputs, inputs, and delays in the model.

A local estimate $\hat{y}$ can be obtained from the solution of the weighted regression problem

$\hat{\beta}=\arg \min _{\beta} \sum_{k=1}^{N} \ell(y(k)-m(\varphi(k), \beta)) \cdot W\left(\frac{\|\varphi(k)-\varphi(t)\|_{M}}{h}\right)$,

where $\ell(\cdot)$ is a scalar-valued and positive norm function, $\|u\|_{M} \triangleq \sqrt{u^{T} M u}$ is a scaled distance function on the regressor space, and $h$ is a bandwidth parameter controlling the size of the local neighborhood, and $W(\cdot)$ is a window function (usually referred to as the kernel) assigning weights to each remote data point according to its distance from $\varphi(t)$. For this work, we choose to work with a tricube kernel.

In principle, it is possible to use any nonlinear model structure as a local model in Equation 6. However, if a quadratic norm, $\ell(\varepsilon)=\varepsilon^{2}$ is used and the model is linear in the unknown parameters, the estimate can be easily computed using simple and powerful least squares methods. We thus assume a local linear model structure,

$$
m(\varphi(k), \beta)=\beta_{0}+\beta_{1}^{T}(\varphi(k)-\varphi(t))
$$

as the default choice in the rest of the paper. If $\hat{\beta}_{0}$ and $\hat{\beta}_{1}$ denote the minimizers of (6) using the model from (7), a one-step ahead prediction of $y(t)$ is given by

$$
\hat{y}(t)=m(\varphi(t), \hat{\beta})=\hat{\beta_{0}} \text {. }
$$

Each local regression problem produces a single prediction $\hat{y}(t)$ corresponding to the current regression vector $\varphi(t)$. To obtain predictions at other locations in the regressor space, the weights change and new optimization problems have to be solved. This is in contrast to the global modeling approach where the model is fitted to data only once and then discarded.

The bandwidth $h$ controls the neighborhood size and has a critical impact on the resulting estimate since it governs a trade-off between the bias and variance errors of the estimate. Traditional bandwidth selectors produce a single global bandwidth; in MoD estimation, bandwidth is computed adaptively at each prediction. While various measures are available for this purpose [13], the method used in this paper is the Akaike's Final Prediction Error (FPE). 


\section{Semi-Physical Modeling}

In system identification, the plant under study may exhibit significant nonlinear behavior, resulting in poor predictive performance by linear models. The model requirements may not necessarily require the time and effort for construction of a first principles model. The user may decide to include regressor terms into the model based on physical insight of the plant. This is the basic concept behind semi-physical modeling (Lindskog [8]). By including nonlinear regressor terms which are linear in the estimated parameters, it may be possible to improve model performance while still using linear least-squares estimation. This leads to a predictor of the form

$$
\hat{y}_{s p}(t)=\theta_{s p}^{T} \varphi(t)
$$

where

$$
\theta_{s p}=\left[\theta_{1} \theta_{2} \ldots \theta_{n}\right]^{T}
$$

While semi-physical modeling and neural network modeling are both powerful approaches by themselves, the combination of the two methods can result in a fit that is better than the original semi-physical model, (Forsell and Lindskog [5]). The semi-physical portion allows the user to include what is already known about the process and help keep the neural network from getting trapped in a local minima. The neural network picks up any additional nonlinearities and noise in the residuals

$$
\varepsilon_{s p}\left(t \mid \hat{\theta}_{s p}\right)=y(t)-\hat{y}_{s p}\left(t \mid \hat{\theta}_{s p}\right)
$$

The architecture of this combination is presented in Figure 2.

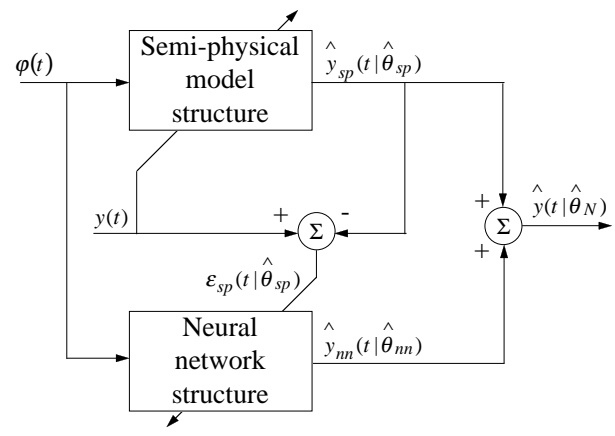

Figure 2: Architecture of semi-physical models combined with neural network.

\section{Case Study}

RTP reactors are playing an increasingly important role in the semiconductor processing industries by offering wafer fabrication operations such as annealing, oxidation and chemical vapor deposition in succession, without having to move the wafer between reactors. Of particular importance in semiconductor processing is the temperature/time profile repeatability and the ability to achieve high rates of temperature increase and decrease, while maintaining temperature uniformity across the diameter of the wafer. Increases of as little as $2 \mathrm{~K} / \mathrm{s}$ in the ramp rate or cool down can reduce the cost of ownership significantly, (Reid and Sitaram [11]). Estimates for acceptable tolerance of temperature control variability for semiconductor processing range from $\pm 12 K$ to $\pm 3 K$, (Ray [10]).

Honeywell Technology Center has developed a simplified model of a RTP wafer reactor, (Christoffel et al. [3]). The reactor consists of five halogen/tungsten lamps placed concentrically on the floor of the reactor. Above the lamps is a quartz window. The wafer to be processed is positioned above the quartz window. The reacting gases are pumped into the reactor through the gas showerhead in the top of the reactor. Figure 3 shows a schematic of the reactor configuration. The 5 inputs to the system are the percent power to each lamp, ranging from 0 to $100 \%$. The 5 outputs of the system are the temperatures $[\mathrm{K}]$ of the wafer directly above the lamps. The simplified model developed by

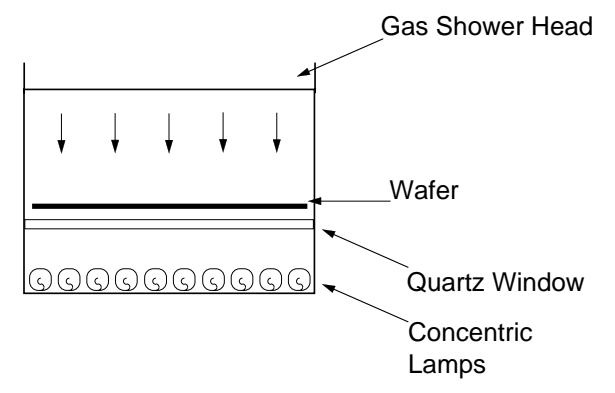

Figure 3: Schematic of RTP reactor configuration

Honeywell is shown in Equation 12. This model, with the parameters $P_{i}$ identified by Honeywell, served as the "truth model" in our study. The quartic terms were included based on the assumption of heat transfer gains and losses due to radiation. The remaining terms are included to provide rough approximations to changes in wafer properties with temperature, most likely of which is emissivity. This model had an RMS error of $1.2 \mathrm{~K}$ in comparison to the true plant. To provide a realistic effect of process noise to the simulation, Gaussian noise with a variance of $4.2 \%$ and a sampling time of 0.2 seconds was added to the percent power input signal of our true plant.

$$
\begin{aligned}
y_{k}= & P_{1} \cdot y_{k-1}+P_{2} \cdot\left(\frac{y_{k-1}}{1000}\right)^{4}+P_{3} \cdot\left(\frac{y_{k-1}}{1000}\right)^{3} \\
& +P_{4} \cdot\left(\frac{y_{k-1}}{1000}\right)^{2}+P_{5} \cdot\left(\frac{u_{k-1}}{100}\right)^{4}+P_{6} \cdot\left(\frac{u_{k-1}}{100}\right)^{3}
\end{aligned}
$$




$$
+P_{7} \cdot\left(\frac{u_{k-1}}{100}\right)^{2}+P_{8} \cdot u_{k-1}+P_{9}
$$

In designing the multi-level pseudo-random sequence
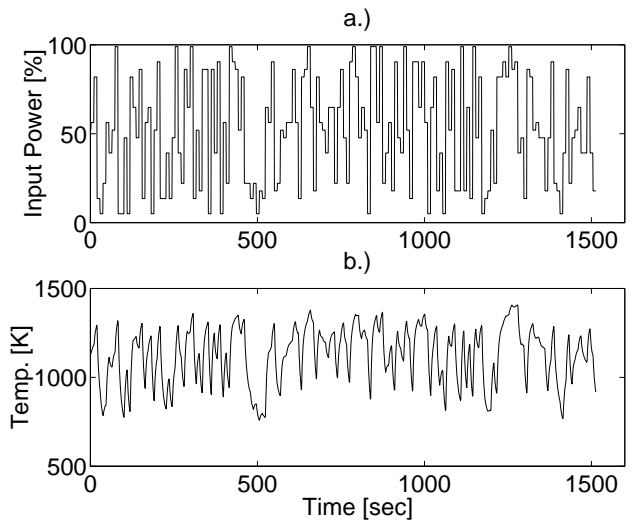

Figure 4: Estimation Data, a.) \% power to the reactor, b.) Temperature of the wafer $[\mathrm{K}]$

data for estimation, we chose to excite the plant between $5 \%$ and $99 \%$ of the lamp power, allowing an output range of temperature between $723 \mathrm{~K}$ and $1469 \mathrm{~K}$. We chose an $\alpha=2$ and $\beta=6$. The dominant time constant range of the RTP simulation was between 7 and 33 seconds. Following the design procedure in Section 2, a 13-level signal, $G F(13)$, was generated with $n_{r}=2$ shift registers, a switching time $T_{s w}$ of 9 seconds and suppression of harmonics $h$ of multiple of 2 . This signal had a length of 1512 seconds. For more details and possible variations on the design, see Braun et al. [2]. The validation data was independently designed, based on temperature ramp rates and processing times normally experienced with this reactor, (Christoffel [3]). The validation data exhibits ramp rates from $27.8 \mathrm{~K} / \mathrm{s}$ to $71.7 \mathrm{~K} / \mathrm{s}$ and processing times from $20 \mathrm{~s}$ to $120 \mathrm{~s}$.

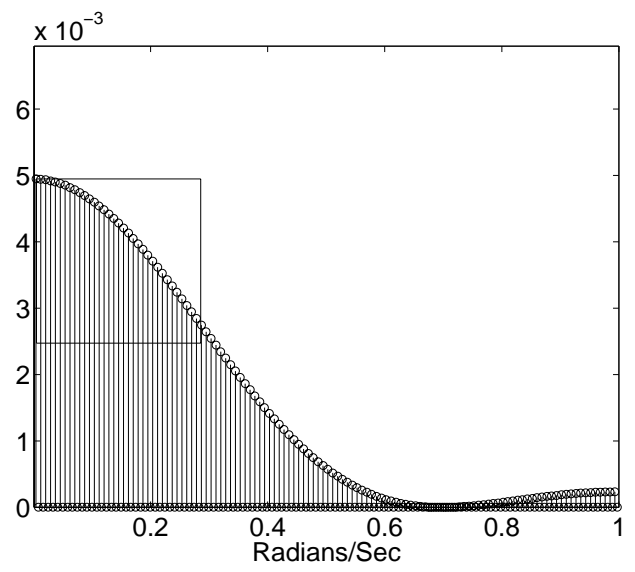

Figure 5: Control relevant region in the power spectrum of a 13-level sequence

For all candidate models, estimation of model parameters was made on only one period of the input signal and model validation was performed on the independent validation data set. The first model that was fit to

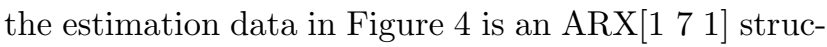
ture. This structure was found to have the lowest RMS error on the validation data set out of a range of ARX structures, $1 \leq n_{a} \leq 10,1 \leq n_{b} \leq 10$ and $1 \leq n_{k} \leq 10$. The semi-physical structure of Equation 12, was also fit to the estimation data to determine how well the "truth-model" structure would fit with the presence of noise. The reduced semi-physical (RSP) structure of Equation 13 was obtained by the assumption that the temperature dependence of the wafer was controlled more by the ability of heat to transfer into the wafer, rather than the energy generated by the lamp. The lower order nonlinearities were eliminated altogether.

$$
\hat{y}_{s p}(k \mid \theta)=\theta_{1} \cdot y_{k-1}+\theta_{2} \cdot\left(\frac{y_{k-1}}{1000}\right)^{4}+\theta_{3} \cdot u_{k-1}+\theta_{4}
$$

To combine the reduced semi-physical structure with a neural network, a feedforward net with 4 hidden neurons and a total of 24 additional parameters was added. We chose to use a sigmoidal basis function $\sigma(\cdot)$. The resulting structure is as follows.

$$
\begin{array}{r}
\hat{y}_{\text {spnn }}(k \mid \theta)=\theta_{s p}^{T} \varphi(k)+\sum_{i=1}^{4} \alpha_{i} \sigma\left(\beta_{i}^{T} \varphi(k)-\gamma_{i}\right) \\
\text { where } \varphi(k)=\left[y_{k-1}\left(\frac{y_{k-1}}{1000}\right)^{4} u_{k-1} 1\right]^{T}
\end{array}
$$

For the MoD predictor, the regressor space which provided the lowest RMS fit on the validation data was used for comparison against the other model structures. A regressor vector of $n_{a}=1, n_{b}=7$, and $n_{k}=1$ was found to provide the best fit. The fits for all of the models are presented in Table 1. These fits can be viewed in Figures 6 and 7. The use of the MoD predictor pro-

Table 1: Error values for model fit on validation data:

RMS MAX

\begin{tabular}{ccc} 
Model $\left(n_{a} n_{b} n_{k}\right)$ & Error $[\mathrm{K}]:$ & Error $[\mathrm{K}]:$ \\
\hline ARX & 37 & 65 \\
RSP & 35 & 62 \\
Semi-physical & 27 & 52 \\
RSP w/ NN & 17 & 52 \\
MoD $(171)$ & 17 & 31 \\
MoD $(111)$ & 21 & 46
\end{tabular}

vides a fit as good as a combined semiphysical/neural network model. The neural network portion required considerable computational effort to determine an initial parameter set and to train the network. While it is desirable to use feasible initial parameters, our software was limited to choosing a set of initial parameters randomly, evaluating each for fit and choosing the best one. The reduced semiphysical model requires engineering effort to choose and evaluate possible regres- 


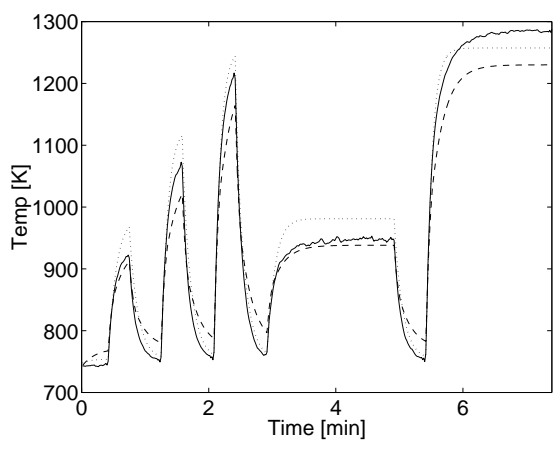

Figure 6: RTP Validation Data, Solid-Plant Output, Dashed-ARX Model, Dotted-Semi-physical Model.

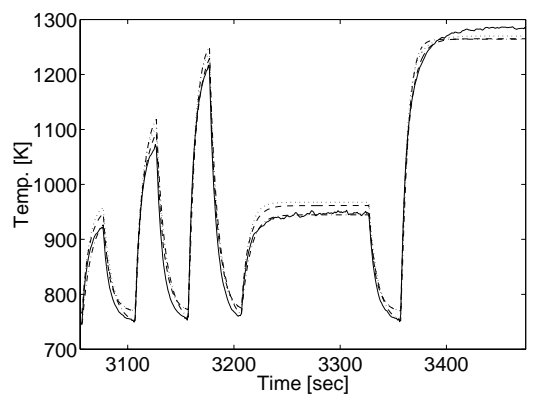

Figure 7: RTP Validation Data, Solid-Plant, DashedMoD predictor $n_{a}=n_{k}=1, n_{b}=7$, DottedMoD predictor $n_{a}=n_{b}=n_{k}=1$, Dash DotRSP w/ Neural Net.

sors. The MoD simulation does not require the significant effort up front, but utilizes system resources for each prediction.

\section{Conclusions}

We have shown how a priori knowledge can be used to efficiently design an input signal and determine simple, but viable candidate models from physical insight for nonlinear plants. It has been demonstrated on the RTP case study that the simulation based on the $\mathrm{MoD}$ estimator rivals that of the combined semiphysi$\mathrm{cal} /$ neural network approach. However, the MoD estimator achieves this level of performance by using less sophisticated structural information.

We would like to thank Peter Lindskog, formerly of Linköping University and presently with Atero AB, for use of his Fuzzymodeling Software for Matlab [9].

\section{References}

[1] H. A. Barker and M. Zhuang. "Design of pseudorandom perturbation signals for frequency-domain identification of nonlinear systems." 11th IFAC Symp. on System Ident., 3, 1997.

[2] M. W. Braun, D. E. Rivera, A. Stenman, W. Foslien, and C. Hrenya. "Multi-level Pseudo-Random Signal Design and "Model-on-Demand" Estimation Applied to Nonlinear Identification of a RTP Wafer Reactor.", In: Proceedings of ACC'99, June 12-14, 1999.

[3] J. Christoffel, W. Foslien, A. Mathur, and M. Ekblad. "Model Based Control and Optimization Techniques from the Advanced Materials Industry Applied to Semiconductor Processes", AEC/APC Workshop VII, pgs. 157-172, November 1995.

[4] G. Cybenko, Just-in-time learning and estimation. In S. Bittani and G. Picci, editors, Identification, Adaptation, Learning, NATO ASI series, pages 423-434, Springer, 1996.

[5] U. Forsell and P. Lindskog. "Combining Semiphysical and Neural Network Modeling: An Example of its Usefulness." In SYSID '97: 11th IFAC Symposium on Sytem Identification. Vol. 2. pp. 795-798, 1997.

[6] K. Godfrey. Perturbation Signals For System Identification. Prentice Hall International (UK) Limited, Hertfordshire UK, 1993.

[7] R. K. Pearson and B. A. Ogunnaike. Nonlinear Process Control. M. A. Henson and D. E. Seborg Editors, Prentice Hall PTR, Upper Saddle River, New Jersey. 1997.

[8] P. Lindskog. "Methods, Algorithms and Tools for System Identification Based on Prior Knowledge." Dissertation No. 436. Department of Electrical Engineering, Linköping University. S-581 83 Linköping, Sweden, 1996.

[9] P. Lindskog. "A System Identification Software Tool for General MISO ARX-type of Model Structures." Technical Report LiTH-ISY-R-1918. Department of Electrical Engineering, Linköping University. S-581 83 Linköping, Sweden, 1996.

[10] A. K. Ray. "RTP Temperature Control Requirement For Submicron Device Fabrication." SPIE - The International Society for Optical Engineering: Rapid Thermal and Laser Processing. Vol. 1804. pp. 2-12, 1993.

[11] K. G. Reid and A. R. Sitaram. "Rapid Thermal Processing for ULSI Applications: An Overview." In Solid State Technology. Vol. 39. pp. 63-74, 1996.

[12] A. Stenman, F. Gustafsson, and L. Ljung. "Just in time models for dynamical systems." In Proceedings of the 35th IEEE Conference on Decision and Control. Kobe, Japan, 1996.

[13] A. Stenman. "Just-in-Time Models with Applications to Dynamical Systems." Licentiate thesis LIUTEK-LIC-1997:02. Department of Electrical Engineering, Linköping University. S-581 83 